
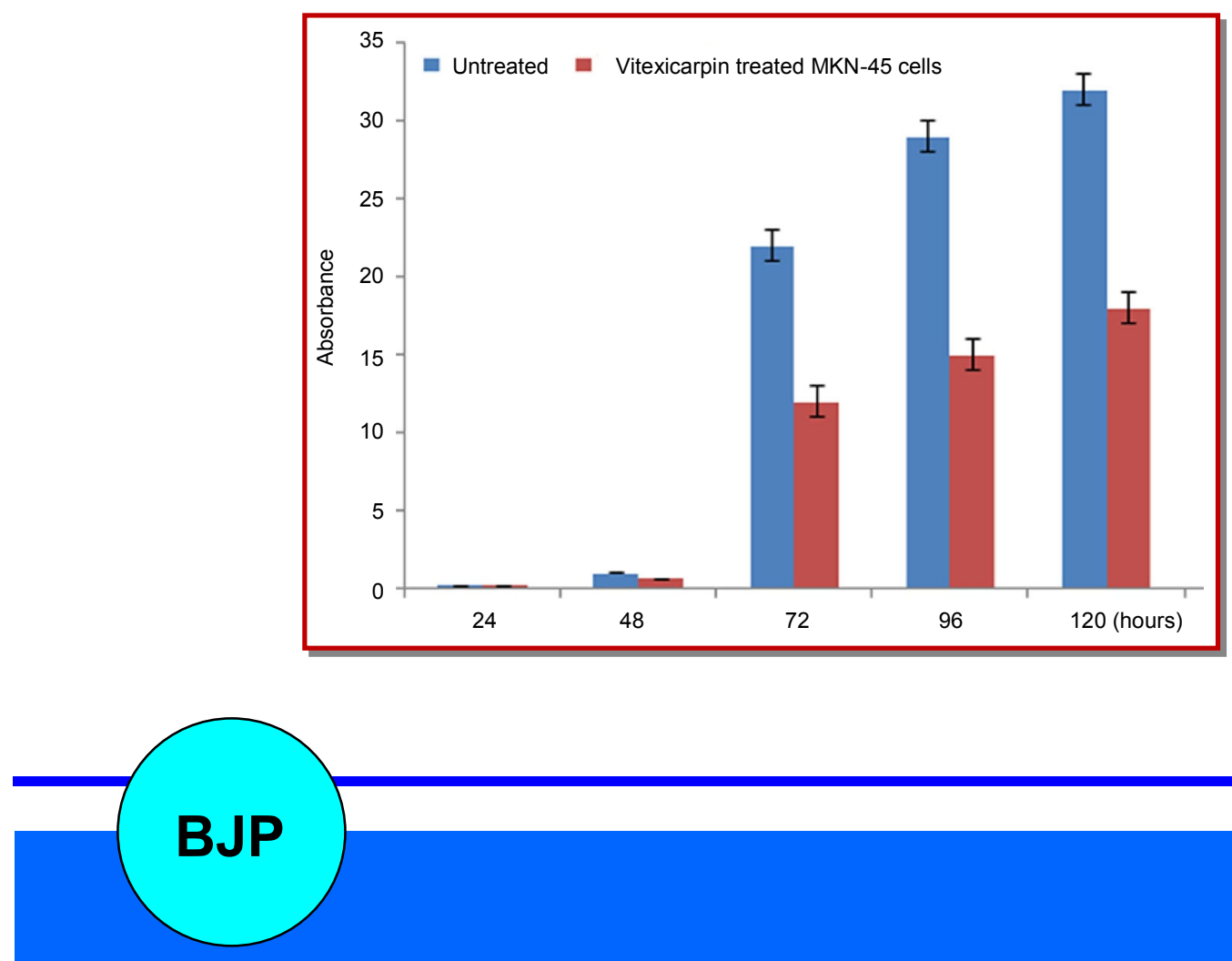

Bangladesh Journal of Pharmacology

Research Article

\title{
Vitexicarpin inhibits overexpression of GNAO1 and plays a role in gastric cancer cell proliferation and apop- tosis
}




\title{
Vitexicarpin inhibits overexpression of GNAO1 and plays a role in gastric cancer cell proliferation and apoptosis
}

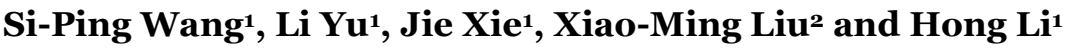 \\ ${ }^{1}$ Domestic Department of Health Management Institute, The Chinese People's Liberation Army General Hospital, \\ Beijing, China; ${ }^{2}$ Key Laboratory of Animal Ecology and Conservation Biology, Institute of Zoology, Chinese \\ Academy of Sciences, Beijing, China.
}

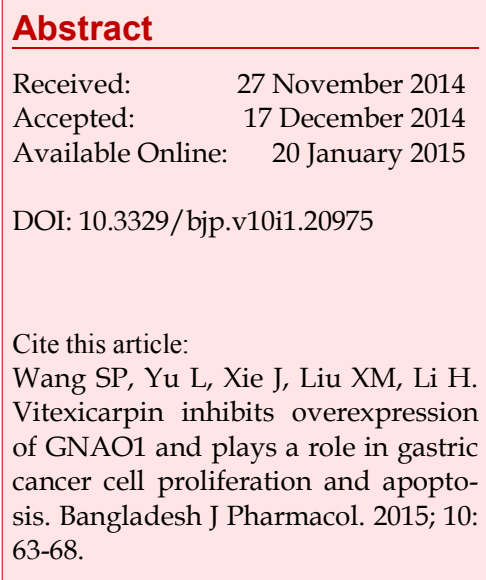

\section{Abstract}

The present study demonstrates the effect of vitexicarpin on down-regulation of GNAO1 in human gastric cancer cell lines. The results from Western blot analysis revealed that vitexicarpin treatment inhibited the GNAO1 expression in MKN-45 cells at a concentration of $30 \mu \mathrm{M}$. Examination of cell proliferation using CCK-8 cell proliferation kit showed $70 \%$ reduction in the vitexicarpin treated cells compared to untreated control cells. Cell cycle analysis using propidium Iodide staining followed by flow cytometry showed cell cycle arrest at G0/G1 with reduction of cell population in the S-phase. There was a significant aneuploidy in the controls compared to zero aneuploidy in the GNAO1 down-regulated cells. Transwell chamber and scratch wound healing assay respectively showed $65 \%$ and $42 \%$ reduction in migration of vitexicarpin treated-MKN-45 cells. Therefore, vitexicarpin treated inhibition of GNAO1 can be a potential therapeutic strategy for the treatment of gastric cancer.

\section{Introduction}

Gastric cancer, the second most frequent cause of cancer deaths (Shibuya et al, 2002), exhibited the stable mortality over the past 20 years, suggesting the urgent need for new therapies (Karpeh et al, 2001). In the United States, the 5-year survival rate for gastric cancer patients is only $5-15 \%$ (Karpeh et al, 2001). The molecular mecha-nisms underlying gastric carcinogenesis are yet fully understood (Peek and Blaser, 2002; Yuasa, 2003).

The alpha subunit of the heterotrimeric guanine nucleotide-binding proteins (Gao) which is encoded by guanine nucleotide-binding protein (G protein), alpha activating activity polypeptide $\mathrm{O}$ (GNAO1) plays a key role in the development of breast and hepatocellular carcinoma. Recently it was reported that silencing of GNAO1 by RNA interference in GC cells markedly inhibited the proliferation of GC cells and promoted apoptosis (Zhen et al., 2013). Therefore, GNAO1 is an important target for the clinical treatment of gastric cancer.

Vitexicarpin (Figure 1), isolated from viticis fructus (Vitex rotundifolia), has long been used as an antiinflammatory herb in traditional Chinese medicine (Choudhary et al., 2009; Mesaik et al., 2009). Vitexicarpinis reported to induce growth inhibition, cell cycle arrest, and apoptosis in human cervical cancer cell line HeLa (Chen et al., 2011; Zeng et al., 2012), hepato-

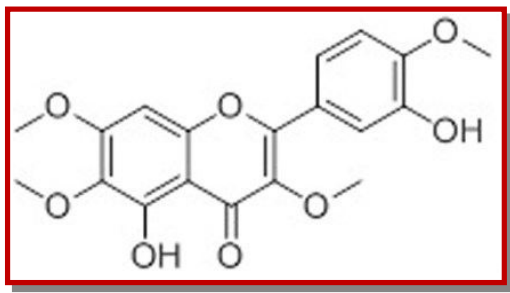

Figure 1: Structure of vitexicarpin 
cellular carcinoma cell line HepG2 (Yang et al., 2011; He et al., 2012), lung epithelial cell line A549 (Koh et al., 2011), and leukemic cell line K562 (Shen et al., 2009). The apoptotic effect of vitexicarpin has been shown to be due to activation of c-Jun N-terminal kinase (JNK) in HeLa cells (Zeng et al., 2012), inactivation NF-KB in A549 cells (Koh et al., 2011), caspase-3 activation in K562 cells (Shen et al., 2009), etc. In the present study, we investigated the effect of vitexicarpin on GNAO1 in human gastric cancer cell line MKN-45.

\section{Materials and Methods}

\section{Cell lines and cell culture}

MKN-45 gastric cell line, were obtained from The Cell Bank of Type Culture Collection of Chinese Academy of Sciences, Shanghai Institute of Cell Biology (China). The cells were maintained in Dulbecco's modified Eagle medium supplemented with $10 \%$ fetal bovine serum containing penicillin 100 units/mL, streptomycin 100 $\mathrm{mg} / \mathrm{mL}$ and $10 \%$ FBS (PAA).

\section{Western blotting}

Total protein was separated on a $12 \%$ SDS-PAGE gel and transferred onto a PVDF membrane blocked in 5\% dried milk for 1 hour at room temperature. The membrane was incubated with specific primary antibody at $4^{\circ} \mathrm{C}$ overnight. After washing thrice with Tris-buffered saline Tween-20 (TBST) membrane was incubated with horseradish peroxidase-conjugated secondary antibody at room temperature for 1 hour. The membrane was washed again with TBST and developed using enhanced chemiluminescence (ECL) (Amersham Life Sciences, Amersham, UK). The staining intensity of the bands was quantified by densitometry, using image analyzing software (Multi Gauge Ver 3.2; Japan).

\section{Cell proliferation assay}

MKN-45 cells were plated at a density of $5 \times 10^{3}$ cells per well in 96-well plates. After culturing for 24 hours, cells were treated with vitexicarpin. Cell proliferation was evaluated at 24, 48, 72 and 96 hours after treatment by determining the number of cells with Cell Proliferation Reagent WST-1 (Roche Diagnostic), as per the instructions manufacturer.

\section{Flow cytometry analysis}

At 24 hours after vitexicarpin treatment, MKN-45 cells were harvested and washed with PBS. The cells were fixed with $70 \%$ ethanol overnight at $-20^{\circ} \mathrm{C}$, washed with $3 \%$ BSA/PBS twice and resuspended in PBS containing $50 \mathrm{mg} / \mathrm{mL}$ propidium iodide and $10 \mathrm{mg} / \mathrm{mL}$ RNase A for $30 \mathrm{~min}$ in dark. Flow cytometry was used for analysis of DNA content and CELL Quest software (Becton Dickinson, USA) analysis of cell-cycle phases.

\section{Scratch wound healing assay}

In 24 well plates, $3 \times 10^{5} \mathrm{MKN}-45$ cells per well were plated and allowed to form $100 \%$ confluence overnight. Using pipette tip the cells wounded carefully and washed with PBS. The cells were continuously cultured in DMEM medium at $37^{\circ} \mathrm{C}$ in $5 \% \quad \mathrm{CO}_{2}$ atmosphere. Inverted fluorescent microscope fitted with a digital camera was used to monitor the rate of wound closer at 0,12 and 24 hours.

\section{Migration assay}

The effect of GNAO1 down-regulation on inhibition of migration potential inMKN-45 cells was determined by using transwell cell culture inserts. $2 \times 10^{5}$ cells were plated in the upper chamber of the inserts containing serum-free DMEM. The lower chamber was filled with DMEM containing 20\% FBS (chemo-attractant). The control wells contained serum-free DMEM in both upper and lower chambers. The wells were incubated at $37^{\circ} \mathrm{C}$ for 20 hours in a humidified $5 \% \mathrm{CO}_{2}$ atmosphere. The plates after fixing and staining were observed under fluorescent microscope.

\section{Cell invasion assay}

The ECM gel (Sigma, USA) coated transwell plates were incubated at $37^{\circ} \mathrm{C}$ for 1 hour to produce an artificial basement membrane.

\section{Statistical analysis}

The data were expressed as the mean \pm standard deviation (S.D.). $\chi^{2}$-test was used to compare the values for the test and control samples. A value of $p<0.05$ was taken as significant. Each assay was performed three times.

\section{Results}

We used Western blot analysis to determine the level of GNAO1 expression in MKN-45 cells. The results revealed that the expression of GNAO1 in vitexicarpin treated-MKN-45 cells was significantly reduced compared to that of untreated MKN-45 cells (Figure 2). The inhibition of GNAO1 expression was significant at 30 $\mu \mathrm{M}$ concentration of vitexicarpin.

The effect of the GNAO1inhibition on the proliferation potential of MKN-45 cells, was determined by using CCK-8 cell proliferation test. The results demonstrated a significant inhibitory effect on cell proliferation in vitexicarpin treated-MKN-45 cells compared to that of the untreated controls (Figure 3). The vitexicarpin treated-MKN-45 curve is seen lagging behind the controls. On day 5 of the assay, the percentage vitexicarpin treated-MKN-45 cells were $70 \%$ of the untreated control cells.

To clarify the mechanisms underlying growth inhibi- 


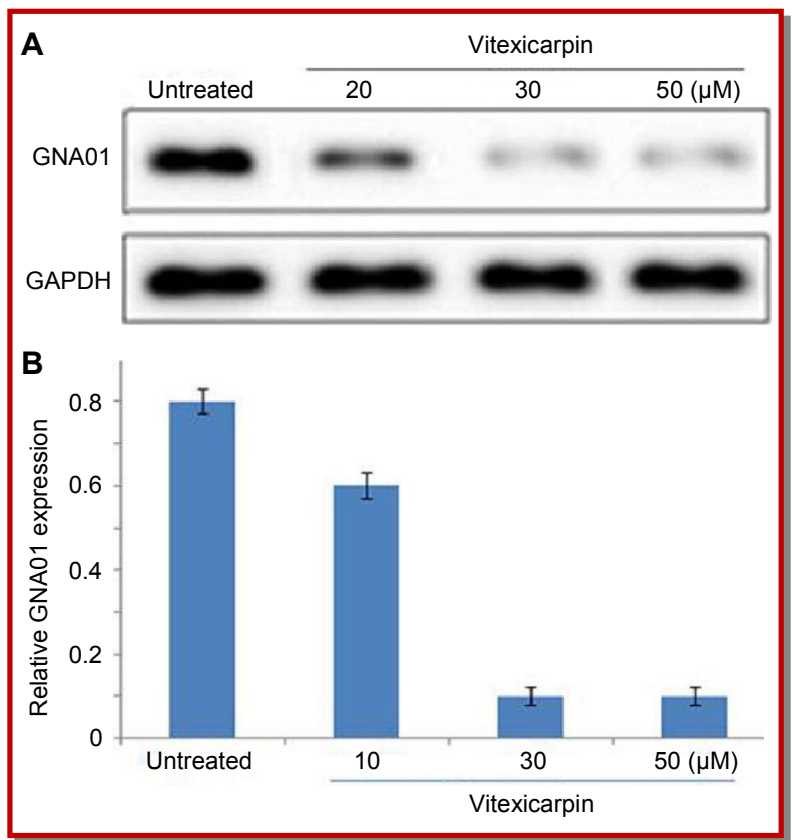

Figure 2: Western Blot analysis of vitexicarpin treated and untreated MKN-45 cells. (A). Western blot image of untreated MKN-45 cells and MKN-45 cells treated with 10, 30, $50 \mu \mathrm{M}$ of vitexicarpin. (B). Quantification of GNAO1 expressions as relative optical densities. The data are the mean \pm SD from three different experiments and the results were statistically significant at $\mathrm{p}<0.05$

tion by GNAO1-down-regulation on MKN-45 gastric cell lines, we performed cell-cycle analysis by flow cytometry on cells stained with propidium iodide. The results showed a decrease in vitexicarpin treated-MKN45 cell population in the S-phase of the cell cycle compared to the untreated control. The cell cycle was arrested at G0/G1 with more than 30\% cell accumulation. There was $15 \%$ aneuploidy in the untreated controls compared to zero aneuploidy in the vitexicarpin treated-MKN-45 cells (Figure 4A, 4B).

We observed a significant difference in the migration and invasion potential in the vitexicarpin treated-MKN45 cells compared to untreated control MKN-45 cells. The results from inverted fluorescent microscopy showed no single cell migrated or invaded through the filter control wells without a chemoattractant. However, we observed a significant migration and invasion in all wells with $20 \%$ FBS as a chemoattractant (Figure $5)$. On the other hand, there was more than $65 \%$ reduction in the migration of vitexicarpin treated-MKN -45 cells compared to the untreated control cells after 20 hours of incubation.

The results, from scratch wound healing assay (Figure 6) revealed near perfect congruity with the transwell migration assays. There again, vitexicarpin treatedMKN-45 cells migrated at a slower rate (42\%) compared to the untreated control cells in terms of the wounded area covered over time. The control groups were covering the wounded area at a rate nearly two-fold that of the vitexicarpin treated-MKN-45. By 24 hours' time, the total wounded area remaining as a percentage of the original area was about $20 \%$ and $45 \%$ for the controls and vitexicarpin treated-MKN-45 cells respectively.

\section{Discussion}

One of the characteristic features of cancer is the uncontrolled cell proliferation induced by mitogenic growth signals (Hanahan and Weinberg 2000; Kops et al., 2005). Silencing of GNAO1 by RNA interference leads to a significant inhibition of proliferation and induction of apoptosis in GC cells (Zhen et al., 2013). Our study demonstrated that down-regulation of GNAO1 in vitexicarpin treated-MKN-45 cells inhibited the cell proliferation compared to that of untreated control cells. Thus GNAO1 down-regulation inhibits the uncontrollable growth in MKN-45 cells. Vitexicarpin treatment also resulted in decrease of cell population in S-phase with simultaneous increase in G0/G1 phase of the cell cycle.

Aneuploidy outcome of the defects in mitotic check points is proportional to genomic instability and severity (Sen, 2000; Duesberg et al., 2000). It is observed in most human cancers (Sen, 2000). We observed that there was about $15 \%$ aneuploidy in untreated control cells compared to that in vitexicarpin treated-cells
Figure 3: Result of cell proliferation assay. It shows the proliferation of vitexicar pin treated-MKN-45 cells lagging behind the untreated control cells. Values represent average of three measurements and the results were statistically significan $(\mathrm{p}<0.05)$ 


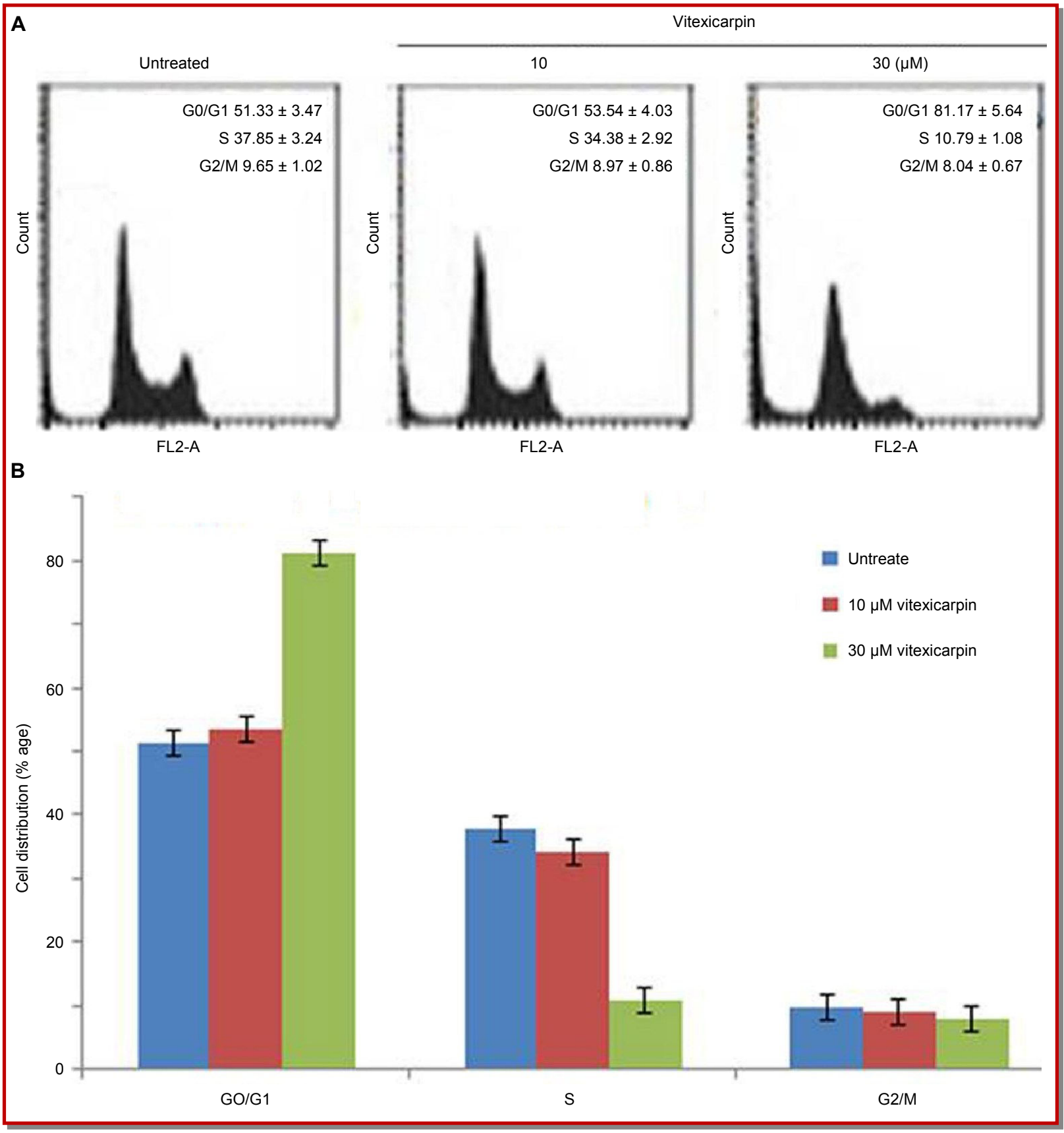

Figure 4: This figure shows cell cycle analysis of MKN-45 cells. (A) MKN-45 cells without vitexicarpin or treated with 10 and 30 $\mu \mathrm{M}$ and (B) cluster bar chart of the cell cycle showing the percentage distributions of the cell cycle phases

with no aneuploidy. A unique property associated with tumor cell metastasis is the invasive and migratory phenotype acquisition along with increased expre-ssion of cell motility related genes (Duesberg and Rasnick, 2000; Chambers et al., 2002; Zhang et al., 2011; Yamaguchi et al., 2005; Fried and Wolf 2003; Wang et al., 2004; Wang et al., 2005). Metastasis is the most common cause of death in cancer (Chambers et al., 2002). The results from our study showed a significant decrease in metathesis in the vitexicarpin treated-MKN45 cells through the trans-well inserts. Therefore, inhibition of GNAO1 expression by vitexicarpin treatment decreased the migration and invasive behavior in gastric cells. Therefore, inhibition of GNAO1 by vitexicarpin treatment may be a promising therapeutic strategy for the treatment of gastric cancer.

\section{References}

Chambers AF, Groom AC, MacDonald IC. Dissemination and growth of cancer cells in metastatic sites. Nat Rev Cancer 


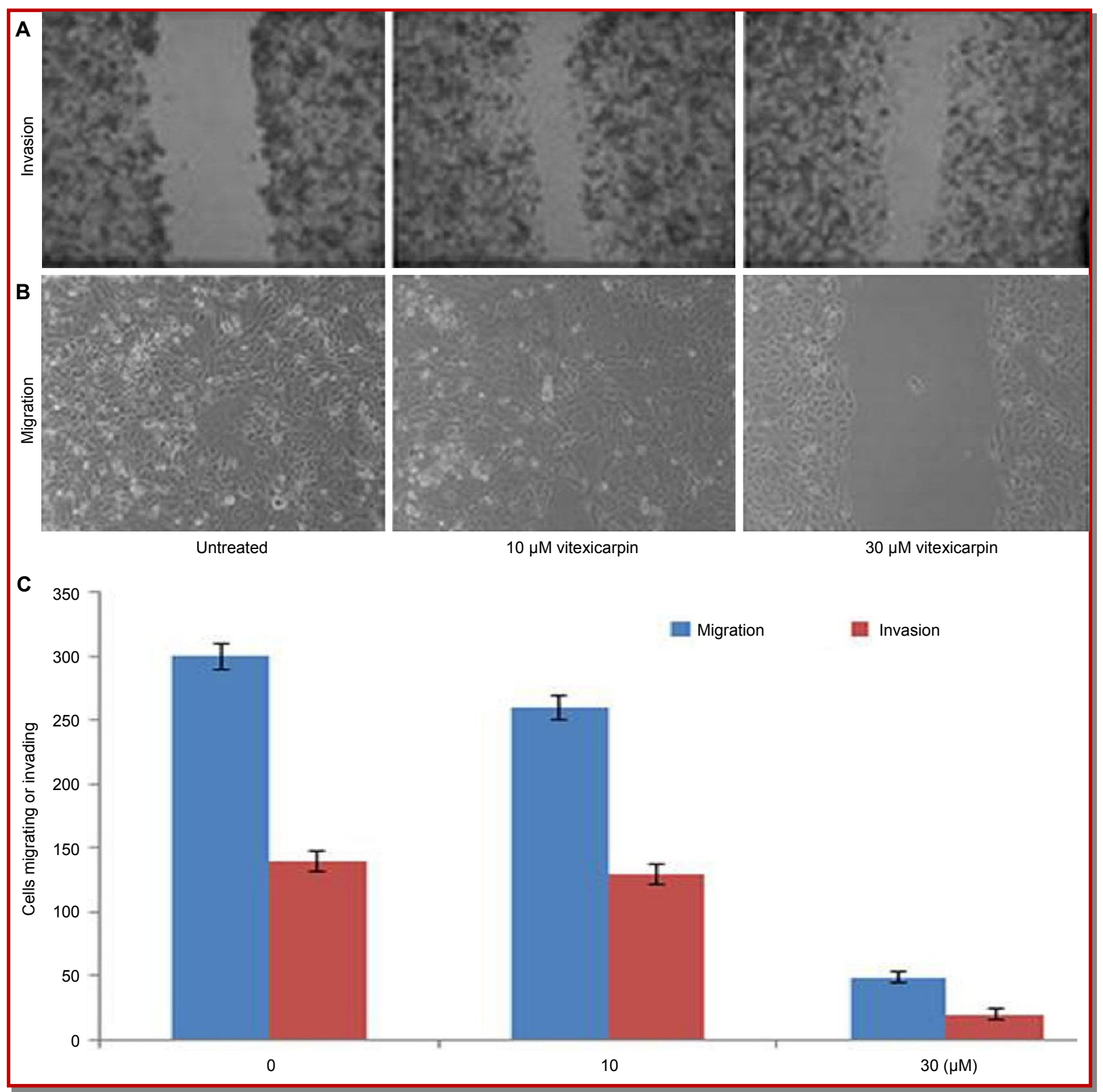

Figure 5: Transwell migration results showing decrease in both migration (B) and invasion (A) in GNAO1 down-regulated cells. Untreated- and vitexicarpin treated-MKN-45 cells. Graphic representation of both migration and invasion (C)

2002; 2: 563-72.

Chen D, Cao J, Tian L, et al. Induction of apoptosis by casticin in cervical cancer cells through reactive oxygen speciesmediated mitochondrial signaling pathways. Oncol Rep. 2011; 26, 1287-94.

Choudhary MI, Azizuddin, Jalil S, et al. Anti-inflammatory and lipoxygenase inhibitory compounds from Vitex agnuscastus. Phytother Res. 2009; 23, 1336-39.

Duesberg P, Rasnick D. Aneuploidy, the somatic mutation that makes cancer a species of its own. Cell Motil Cytoskeleton. 2000; 47: 81-107.

Fried P, Wolf K. Tumour-cell invasion and migration: Diversity and escape mechanisms. Nat Rev Cancer. 2003; 3: 362-74.
Hanahan D, Weinberg RA. The hallmarks of cancer. Cell 2000; 100: 57-70.

He L, Yang X, Cao X, et al. Casticin induces growth suppression and cell cycle arrest through activation of FOXO3a in hepatocellular carcinoma. Oncol Rep. 2012; 29, 103-08.

Karpeh MS, Kelsen DP, Tepper JE. Cancer of the stomach. In: Cancer: Principles and practice of oncology. DeVita VT, Hellman S, Rosenberg SA (eds). 2001, pp 1092-126.

Koh DJ, Ahn HS, Chung HS, et al. Inhibitory effects of casticin on migration of eosinophil and expression of chemokines and adhesion molecules in A549 lung epithelial cells via NFкB inactivation. J Ethnopharmacol. 2011; 136, 399-405.

Kops GJ, Weaver BA, Cleveland DW. On the road to cancer: 


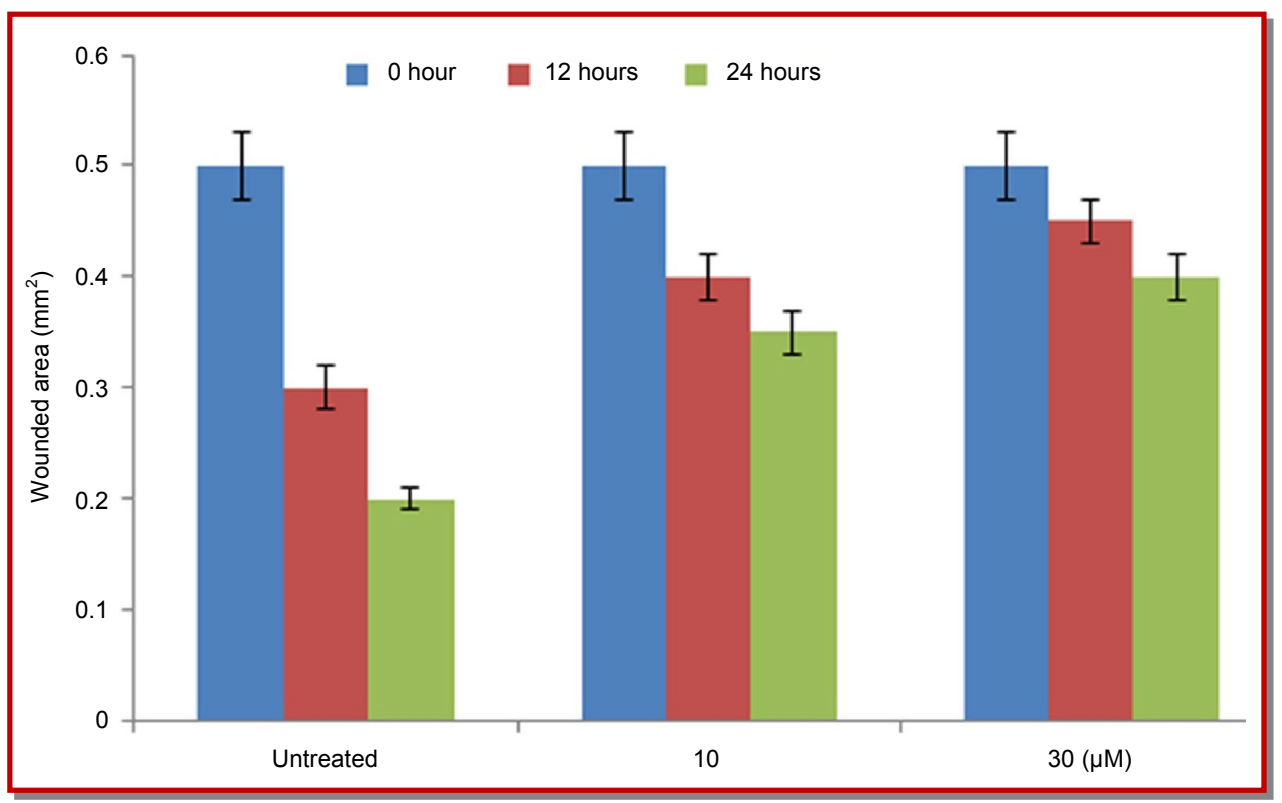

Figure 6: Scratch wound healing assay. Changes in the wounded area remaining with time. Results represent mean of three values and were statistically significant $(\mathrm{p}<0.05)$

Aneuploidy and the mitotic checkpoint. Nat Rev Cancer. 2005; 5: 773-85.

Mesaik MA, Azizuddin, Murad S, et al. Choudhary MI. Isolation and immunomodulatory properties of a flavonoid, casticin from Vitex agnus-castus. Phytother Res. 2009; 23: 1516-20.

Peek Jr RM, Blaser MJ. Helicobacter pylori and gastrointestinal tract adenocarcinomas. Nat Rev Cancer. 2002; 2: 28-37.

Sen S. Aneuploidy and cancer. Curr Opin Oncol. 2000; 12: 8288.

Shen JK, Du HP, Yang M, et al. Casticin induces leukemic cell death through apoptosis and mitotic catastrophe. Ann Hematol. 2009; 88: 743-52.

Shibuya K, Mathers CD, Boschi-Pinto C, Lopez AD, Murray CJ. Global and regional estimates of cancer mortality and incidence by site: II. Results for the global burden of disease 2000. BMC Cancer. 2002; 2: 37.

Wang W, Goswami S, Lapidus K, Wells AL, Wyckoff JB, Sahai $\mathrm{E}$, et al. Identification and testing of a gene expression signature of invasive carcinoma cells within primary mammary tumors. Cancer Res. 2004; 64: 8585-94.
Wang W, Goswami S, Sahai E, Wyckoff JB, Segall JE, Condeelis JS. Tumor cells caught in the act of invading: Their strategy for enhanced cell motility. Trends Cell Biol. 2005; 15: 138-45.

Yamaguchi H, Wyckoff J, Condeelis J. Cell migration in tumors. Curr Opin Cell Biol. 2005; 17: 559-64.

Yang J, Yang Y, Tian L, et al. Casticin-induced apoptosis involves death receptor up-regulation in hepatocellular carcinoma cells. World J Gastroenterol. 2011; 17: 4298-307.

Yuasa Y. Control of gut differentiation and intestinal-type gastric carcinogenesis. Nat Rev Cancer. 2003; 3: 592-600.

Zeng F, Tian L, Liu F, et al. Induction of apoptosis by casticin in cervical cancer cells: Reactive oxygen species dependent sustained activation of Jun N-terminal kinase. Acta Bioch Bioph Sin. 2012; 44: 442-49.

Zhang YH, Wang SQ, Sun CR, Wang M, Wang B, Tang JW. Inhibition of JNK1 expression decreases migration and invasion of mouse hepatocarcinoma cell line in vitro. Med Oncol. 2011; 28: 966-72.

Zhen L, Jun Z, Lijun W, Jie L, Mingqing Z. Overexpression of GNAO1 correlates with poor prognosis in patients with gastric cancer and plays a role in gastric cancer cell proliferation and apoptosis. Int J Mol Med. 2013; 33: 589-96.

\footnotetext{
Author Info

Hong Li (Principal contact)

( e-mail: lihong86010@gmail.com
} 